\title{
Next-generation sequencing study reveals the broader variant spectrum of hereditary spastic paraplegia and related phenotypes
}

\author{
Ewelina Elert-Dobkowska ${ }^{1} \cdot$ Iwona Stepniak ${ }^{1} \cdot$ Wioletta Krysa ${ }^{1} \cdot$ Karolina Ziora-Jakutowicz $^{1} \cdot$ Maria Rakowicz $^{2}$. \\ Anna Sobanska ${ }^{2}$. Jacek Pilch ${ }^{3}$ • Dorota Antczak-Marach ${ }^{4}$. Jacek Zaremba ${ }^{1,5}$ • Anna Sulek ${ }^{1}$ (D)
}

Received: 25 October 2018 / Accepted: 11 January 2019 /Published online: 19 February 2019

(C) The Author(s) 2019

\begin{abstract}
Hereditary spastic paraplegias (HSPs) are clinically and genetically heterogeneous neurodegenerative disorders. Numerous genes linked to HSPs, overlapping phenotypes between HSP subtypes and other neurodegenerative disorders and the HSPs' dual mode of inheritance (both dominant and recessive) make the genetic diagnosis of HSPs complex and difficult. Out of the original HSP cohort comprising 306 index cases (familial and isolated) who had been tested according to "traditional workflow/guidelines" by Multiplex Ligation-dependent Probe Amplification (MLPA) and Sanger sequencing, 30 unrelated patients (all familial cases) with unsolved genetic diagnoses were tested using next-generation sequencing (NGS). One hundred thirty-two genes associated with spastic paraplegias, hereditary ataxias and related movement disorders were analysed using the Illumina TruSight ${ }^{\mathrm{TM}}$ One Sequencing Panel. The targeted NGS data showed pathogenic variants, likely pathogenic variants and those of uncertain significance (VUS) in the following genes: SPAST (spastin, SPG4), ATL1 (atlastin 1, SPG3), WASHC5 (SPG8), KIF5A (SPG10), KIF1A (SPG30), SPG11 (spatacsin), CYP27A1, SETX and ITPR1. Out of the nine genes mentioned above, three have not been directly associated with the HSP phenotype to date. Considering the phenotypic overlap and joint cellular pathways of the HSP, spinocerebellar ataxia (SCA) and amyotrophic lateral sclerosis (ALS) genes, our findings provide further evidence that common genetic testing may improve the diagnostics of movement disorders with a spectrum of ataxia-spasticity signs.
\end{abstract}

Keywords Ataxia-spasticity $\cdot$ Hereditary spastic paraplegia $\cdot$ Movement disorders $\cdot$ Next-generation sequencing.

Electronic supplementary material The online version of this article (https://doi.org/10.1007/s10048-019-00565-6) contains supplementary material, which is available to authorized users.

Anna Sulek

suleka@ipin.edu.pl; ania1sulek@gmail.com

1 Department of Genetics, Institute of Psychiatry and Neurology, Sobieskiego 9 Street, 02-957 Warsaw, Poland

2 Department of Clinical Neurophysiology, Institute of Psychiatry and Neurology, Warsaw, Poland

3 Department of Paediatric Neurology, Medical University of Silesia, Katowice, Poland

4 Clinic of Neurology of Children and Adolescents, Institute of Mother and Child, Warsaw, Poland

5 Division Five of Medical Sciences, Polish Academy of Science, Warsaw, Poland

\section{Introduction}

Hereditary spastic paraplegias (HSPs) comprise a group of genetic disorders resulting from neurodegeneration of the corticospinal tracts. The HSPs' main clinical feature is a progressive spasticity and weakness of the lower limbs. HSP is classified as a pure form when symptoms are limited to: progressive spasticity and weakness of the lower limbs, bladder dysfunction and mild somatosensory deficits. In case of any additional neurological symptoms, a complicated HSP form is recognised. To date, over 70 different SPG loci have been identified, and over 60 corresponding genes have been investigated [1-3]. All modes of HSP inheritance have already been described: autosomal dominant (ADHSP), autosomal recessive (ARHSP), X-linked (XLHSP) and less frequently, mitochondrial. Among 20 different ADHSP subtypes, SPG4 is the most common one, accounting for approximately $40 \%$ of the cases. The frequency of other ADHSP subtypes ranges from $1 \%$ to $10 \%$. The main ARHSPs identified to date are SPG5, SPG7, SPG11 and SPG15 [4]. 
According to population studies, the proportion of families without genetic diagnosis ranged from $45 \%$ to $67 \%$ in the ADHSP and from $71 \%$ to $82 \%$ in the ARHSP groups [5]. Recently reported dual-transmission of some HSP subtypes makes their molecular characterisation even more complicated. Due to the HSP heterogeneity, next-generation sequencing (NGS) became a highly useful screening tool in HSP investigations and differential diagnosis. Broad NGS studies have revealed a clinical and genetic overlap between different HSP subtypes, as well as between other neurodegenerative disorders, such as hereditary spinocerebellar ataxias (SCAs), amyotrophic lateral sclerosis (ALS) and neuropathies [6].

In the present study, we analysed familial HSP patients through spastic-ataxia spectrum disease genes according to the approach suggested by Synofzik et al. [6].

\section{Materials and methods}

The study was approved by the Bioethics Committee of the Institute of Psychiatry and Neurology in Warsaw. All of the participants provided informed consent.

In the presented study, we aimed to test a group of 30 unrelated hereditary spastic paraplegia patients using the targeted Illumina TruSight ${ }^{\text {TM }}$ One Sequencing Panel (Illumina). The original HSP cohort comprised 306 probands in which Multiplex Ligation-dependent Probe Amplification (MLPA) and Sanger Sequencing had been performed to diagnose five HSP subtypes (SPG3, SPG4, SPG6, SPG11 and SPG31) in 62 families [7-10]. Out of the remaining 244 probands, 30 familial HSP index cases were selected for NGS testing. The major inclusion criteria comprise: (i) spastic paraplegia as a main clinical feature, (ii) positive family history and (iii) availability of DNA sample for more than one affected family member and/or potential carriers. The families' history suggested AD inheritance in 18 and AR in 12 families. In three probands, SPG11 deletions and duplication had been identified in one allele, and NGS sequencing focused on searching for the second causative variant to confirm the AR SPG11. One identified carrier of the SPAST pathogenic variant was used as a positive control in the NGS screening (Fig. 1).

All studied patients were evaluated according to the Fink criteria for HSP [11]. The HSP pure form was observed in 16 probands, and the complicated form was observed in 14 probands.

The Illumina TruSight ${ }^{\mathrm{TM}}$ One Sequencing Panel covering the coding regions of the 4813 genes associated with the known clinical phenotypes was used (https://www. illumina.com/products/by-type/clinical-research-products/ trusight-one.html). The panel includes over 125,000 80-mer probes constructed according to the human NCBI37/hg19 reference genome. The probe set was designed for enrichment of approximately 62,000 exons spanning 4813 genes (https://www.illumina.com/products/by-type/clinicalresearch-products/trusight-one.html). The library preparation, labelling and enrichment were performed according to the protocol using $50 \mathrm{ng}$ of DNA input. The coding regions of 132 genes linked to spastic paraplegias, hereditary ataxias and related movement disorders were analysed. The data were analysed using Illumina VariantStudio 2.2 and visualised in Integrated Genomics Viewer (IGV) (Broad Institute). To investigate the evolutionary conservation score (PhyloP) and functional prediction of identified mutations, we used SIFT (http://sift.jcvi. org/), Polyphen2 (http://genetics.bwh.harvard.edu/pph2/), MutationTaster (http://www.mutationtaster.org/) and Alamut software (http://www.interactive-biosoftware.com/), as well as the dbSNP (https://www.ncbi.nlm.nih.gov/ projects/SNP/) and ClinVar databases (https://www.ncbi. nlm.nih.gov/clinvar/).

NGS data were filtered according to the following criteria: (i) read depth higher than 20 reads and variant frequency higher than 25\%; (ii) variants reported less frequently than 0.005 in the Exome Aggregation Consortium database (http://exac.broadinstitute.org/); and (iii) exclusion of all the synonymous and deep intronic variants.

The bioinformatically analysed 132 ataxia-spasticity panel genes involved the following: (1) 37 genes directly linked with HSP: 12-ADHSP, 22-ARHSP and 3-XLHSP; (2) 25 genes linked with hereditary ataxias: $12 \mathrm{AD}$ spinocerebellar ataxia (SCA), 11 ARSCA (SCAR) and four spastic-ataxia (SPAX) genes; (3) three leucodystrophy genes; (4) 14 amyotrophic lateral sclerosis (ALS) genes; (5) 16 genes linked with different neuropathies, including five hereditary motor neuropathies $(\mathrm{HMN})$ and six Charcot Marie-Tooth neuropathies; and (6) other complex movement or multisystem disorders with prominent gait disturbances, comprising 42 genes (Supplementary Table 1). Because certain genes are linked with more than one phenotype, the number of genes and conditions are not equal. The classification and interpretation of the identified variants were performed according to recommendations of the American College of Medical Genetics and Genomic and the Association for Molecular Pathology (ACMGG\&AMP) (Table 1) [12]. Variants selected through filtering were confirmed by Sanger sequencing in the probands and their family members.

\section{Results}

The NGS TruSight ${ }^{\mathrm{TM}}$ One output data reached approximately $97 \%$ of the aligned reads. A mean number of $16,752,119$ reads with 259 base pair length fragments per sample was obtained. An average of $91.2 \%$ of targeted reads passed the Q score, whereas $88 \%$ were covered at least 30 times. 




Fig. 1 Analysed cohort and methods used during HSP diagnostics. Detailed description of the identified variants is presented in tables

In this study, we identified 18 pathogenic and likely pathogenic variants in 16 spastic paraplegia probands, as well as six variants of uncertain significance (Table 2; Table 3). The most frequent HSP genetic types, SPG4 and SPG3, were identified in five probands: SPAST (SPG4) pathogenic variants in three probands and ATL1 (SPG3) in two probands. In four of the mentioned probands, a previous study involved only the MLPA screening, and one of the SPG4 patients was known to carry a pathogenic variant. In 11 out of 22 individuals, in whom SPAST, ATL1 and REEP1 gene single nucleotide variants (SNV) were previously excluded by Sanger sequencing, we identified three HSP subtypes with AD transmission: WASHC5 (SPG8), KIF5A (SPG10) and KIF1A (SPG30) and SPG11 (SPG11) as the only ARHSPs. Moreover, in one case, a homozygous variant in the CYP27Al gene, known as pathogenic in cerebrotendinous xanthomatosis (CTX), was identified. Among six variants of uncertain significance we detected: WASHC5, KIF5A, SETX and ITPRI variants in families with $\mathrm{AD}$ mode of inheritance. We were not able to detect any variant corresponding to phenotype in $27 \%$ of the examined cohort (four cases with $\mathrm{AD}$ and four with AR mode of inheritance).

\section{Autosomal dominant HSPs}

\section{ATL1 (SPG3)}

One known pathogenic ATL1 variant: c. $715 \mathrm{C}>\mathrm{T}$ (p.Arg239Cys) and one novel: c.1064A $>$ C (p.Asn355Thr) were identified in two HSP probands. The variants presented pure HSP with the age of onset at the first and second years of life.

\section{SPAST (SPG4)}

In the SPAST gene, the variants were identified in three probands: a missense (c.1378C >T-p.Arg460Cys), nonsense (c.1597G $>$ T-p.Glu533*) and splice site (c.1617-2A>G) mutation. SPAST c. $1378 \mathrm{C}>\mathrm{T}$ is a known pathogenic variant, a moderately conserved nucleotide and highly conserved amino acid position. The two other SPAST gene variants (c.1597G $>$ T and c. 1617-2A>G) have not been previously described, neither in the patient cohorts nor in population studies. The ages at onset in the three SPG4 patients were 35, 42 and 28 years, 
Table 1 Interpretation of all variants identified in HSP probands according to the ACMGG\&AMP guidelines [Richards and others 2015]

\begin{tabular}{|c|c|c|c|c|}
\hline Patient ID & Gene & cDNA change & ACMG criteria & ACMG classification \\
\hline SPG0902 & ATL1 & $\begin{array}{l}\text { NM_015915.4:c.715C }>\mathrm{T} \\
\text { NP_056999.2:p.(Arg239Cys) }\end{array}$ & $\mathrm{PM} 1+\mathrm{PM} 2+\mathrm{PP} 1+\mathrm{PP} 3+\mathrm{PP} 4+\mathrm{PP} 5$ & Likely pathogenic \\
\hline SPG0901 & ATL1 & $\begin{array}{l}\text { NM_015915.4:c.1064A>C } \\
\text { NP_056999.2:p.(Asn355Thr) }\end{array}$ & $\mathrm{PM} 1+\mathrm{PM} 2+\mathrm{PP} 3+\mathrm{PP} 4$ & Likely pathogenic \\
\hline SPG1301 & SPAST & $\begin{array}{l}\text { NM_014946.3:c.1378C }>\mathrm{T} \\
\text { NP_055761.2:p.(Arg460Cys) }\end{array}$ & $\mathrm{PM} 1+\mathrm{PM} 2+\mathrm{PP} 4+\mathrm{PP} 3+\mathrm{PP} 5$ & Likely pathogenic \\
\hline SPG0102 & SPAST & $\begin{array}{l}\text { NM_014946.3:c.1597G >T } \\
\text { NP_055761.2:p.(Glu533*) }\end{array}$ & $\mathrm{PVS} 1+\mathrm{PM} 2+\mathrm{PM} 4$ + PM5 + PP4 & Pathogenic \\
\hline SPG1401 & SPAST & NM__ $014946.3: c .1617-2 A>G$ & $\mathrm{PVS} 1+\mathrm{PM} 2+\mathrm{PP} 4$ & Pathogenic \\
\hline SPG0403 & WASHC5 & $\begin{array}{l}\text { NM_014846.3:c.647C }>\text { T } \\
\text { NP_055661.3:p.(Pro216Leu) }\end{array}$ & $\mathrm{PP} 1+\mathrm{PP} 3+\mathrm{PP} 4$ & Uncertain significance \\
\hline SPG0302 & WASHC5 & $\begin{array}{l}\text { NM_014846.3:c.1859T>C } \\
\text { NP_055661.3:p.(Val620Ala) }\end{array}$ & $\mathrm{PM} 2+\mathrm{PP} 1+\mathrm{PP} 3+\mathrm{PP} 4+\mathrm{PP} 5$ & Likely pathogenic \\
\hline SPG0201 & KIF5A & $\begin{array}{l}\text { NM_004984.2:c.484C }>\text { T } \\
\text { NP_004975.2:p.(Arg162Trp) }\end{array}$ & $\mathrm{PM} 1+\mathrm{PP} 3+\mathrm{PP} 4+\mathrm{PP} 5$ & Likely pathogenic \\
\hline SPG1402 & KIF5A & $\begin{array}{l}\text { NM_004984.2:c.1402C>T } \\
\text { NP_004975.2:p.(Arg468Trp) }\end{array}$ & $\mathrm{PP} 3+\mathrm{PP} 4$ & Uncertain significance \\
\hline SPG1101 & KIF1A & $\begin{array}{l}\text { NM_001244008.1:c.962G>A } \\
\text { NP_001230937.1:p.(Gly321Asp) }\end{array}$ & $\mathrm{PM} 1+\mathrm{PM} 2+\mathrm{PM} 4+\mathrm{PP} 3+\mathrm{PP} 4$ & Likely pathogenic \\
\hline \multirow[t]{2}{*}{ SPG0601 } & $S P G 11$ & $\begin{array}{l}\text { NM_-025137.3:c.408_428del } \\
\text { NP_079413.3:p. } \\
\quad \text { (Glu136_lle143del) }\end{array}$ & $\mathrm{PM} 2+\mathrm{PM} 4+\mathrm{PP} 4+\mathrm{PP} 5$ & Likely pathogenic \\
\hline & & $\begin{array}{l}\text { NM_025137.3:c.3075insA } \\
\text { NP_079413.3:p.(Glu1026Argfs*4) }\end{array}$ & $\mathrm{PVS} 1+\mathrm{PM} 2+\mathrm{PP} 5$ & Pathogenic \\
\hline \multirow[t]{2}{*}{ SPG1002 } & SPG11 & $\begin{array}{l}\text { NM_025137.3:c.733_734del } \\
\text { NP_079413.3:p.(Met245Valfs*2) } \\
\text { NM_025137.3:c.1471_1472del } \\
\text { NP_- } \\
\quad \text { 079413.3:p.(Leu491_Aspfs*66) }\end{array}$ & $\begin{array}{l}\mathrm{PVS} 1+\mathrm{PM} 2+\mathrm{PM} 3+\mathrm{PP} 5 \\
\mathrm{PVS} 1+\mathrm{PM} 2+\mathrm{PM} 3+\mathrm{PP} 5\end{array}$ & $\begin{array}{l}\text { Pathogenic } \\
\text { Pathogenic }\end{array}$ \\
\hline & & $\begin{array}{l}\text { NM_025137.3:c.6632G >A } \\
\text { NP_079413.3:p.(Arg2211His) }\end{array}$ & PP2 & Uncertain significance \\
\hline \multirow[t]{2}{*}{ SPG1003 } & $S P G 11$ & $\begin{array}{l}\text { NM_E_025137.3:c.1471_1472del } \\
\text { NP_- } \\
\quad \text { 079413.3:p.(Leu491_Aspfs*66) }\end{array}$ & $\mathrm{PVS} 1+\mathrm{PM} 2+\mathrm{PM} 3+\mathrm{PP} 5$ & Pathogenic \\
\hline & & $\begin{array}{l}\text { NM_025137.3:c.3075insA } \\
\text { NP_079413.3:p.(Glu1026Argfs*4) }\end{array}$ & $\mathrm{PVS} 1+\mathrm{PM} 2+\mathrm{PM} 3+\mathrm{PP} 5$ & Pathogenic \\
\hline SPG0702 & SPG11 & $\begin{array}{l}\text { NM_025137.3:c.1275insA } \\
\text { NP_079413.3:p.(Glu426Argfs*3) }\end{array}$ & $\mathrm{PVS} 1+\mathrm{PM} 2+\mathrm{PP} 4$ & Pathogenic \\
\hline SPG0502 & $S P G 11$ & $\begin{array}{l}\text { NM_025137.3:c. } 1457-2 \mathrm{~A}>\mathrm{G} \\
\mathrm{NM} \text { _025137.3:c.5623C>T } \\
\text { NP_079413.3:p.(Gln1875*) }\end{array}$ & $\begin{array}{l}\mathrm{PVS} 1+\mathrm{PM} 2+\mathrm{PM} 3+\mathrm{PP} 5 \\
\mathrm{PVS} 1+\mathrm{PM} 2+\mathrm{PM} 3+\mathrm{PP} 5\end{array}$ & $\begin{array}{l}\text { Pathogenic } \\
\text { Pathogenic }\end{array}$ \\
\hline SPG0301 & $S P G 11$ & $\begin{array}{l}\text { NM_025137.3:c.2849delT } \\
\text { NP_079413.3:p.(Leu950Trpfs*13) }\end{array}$ & PVS1 + PM2 + PM4 & Pathogenic \\
\hline SPG0103 & $S P G 11$ & NM_025137.3:c.2987_2989del & $\mathrm{PM} 2+\mathrm{PM} 4+\mathrm{PP} 3+\mathrm{PP} 4$ & Likely pathogenic \\
\hline SPG0701 & CYP27A1 & $\begin{array}{l}\text { NM_000784.3:c.379C>T } \\
\text { NP_000775.1:p.(Arg127Trp) }\end{array}$ & $\mathrm{PM} 2+\mathrm{PM} 3+\mathrm{PP} 3+\mathrm{PP} 5$ & Likely pathogenic \\
\hline SPG0303 & ITPRI & $\begin{array}{l}\text { NM_001168272.1:c.2687C>T } \\
\text { NP_001161744.1:p.(Ala896Val) }\end{array}$ & $\mathrm{PP} 1+\mathrm{PP} 3$ & Uncertain significance \\
\hline SPG0401 & ITPRI & $\begin{array}{l}\text { NM_001168272.1:c.2687C>T } \\
\text { NP_001161744.1:p.(Ala896Val) }\end{array}$ & $\mathrm{PP} 1+\mathrm{PP} 3$ & Uncertain significance \\
\hline SPG1203 & ITPRI & $\begin{array}{l}\text { NM_001168272.1:c.3412A>G } \\
\text { NP_001161744.1:p.(Met1138Val) } \\
\text { NM_001168272.1c.6304G>T } \\
\text { NP_001161744.1:p.(Ala2102Ser) }\end{array}$ & $\begin{array}{l}\text { PP3 } \\
\text { PP3 }\end{array}$ & $\begin{array}{l}\text { Uncertain significance } \\
\text { Uncertain significance }\end{array}$ \\
\hline SPG0503 & SETX & $\begin{array}{l}\text { NM_015046.5:c.7417C }>\mathrm{G} \\
\text { NP_055861.3:p.(Leu2473Val) }\end{array}$ & $\mathrm{PP} 1+\mathrm{PP} 3$ & Uncertain significance \\
\hline
\end{tabular}

$P V S$ very strong evidence of pathogenicity, $P S$ strong evidence of pathogenicity, $P M$ moderate evidence of pathogenicity, $P P$ supporting evidence of pathogenicity 


\begin{tabular}{|c|c|c|c|c|c|c|c|c|c|c|c|c|c|c|c|c|c|c|c|c|}
\hline 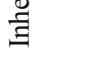 & 主 & 安 & \& & 安 & 安 & \& & \& & 完 & $\stackrel{2}{4}$ & & $\stackrel{\gtrless}{<}$ & & & $\stackrel{ }{<}$ & & $\stackrel{\gtrless}{4}$ & & $\stackrel{2}{4}$ & & $\stackrel{\nwarrow}{4}$ \\
\hline 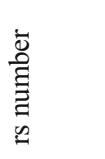 & 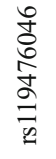 & $\dddot{g}$ & 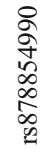 & $\dddot{g}$ & $\cong$ & 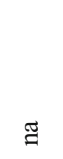 & 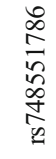 & $\dddot{g}$ & 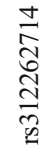 & 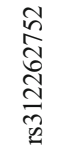 &  & 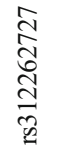 & 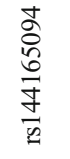 & 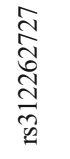 & 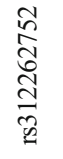 & $\mathscr{Z}$ & & 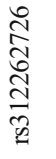 &  &  \\
\hline 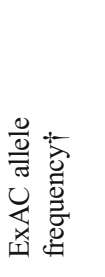 & 0 & 0 & 0 & 0 & 0 & 0 & $\begin{array}{l}\widehat{\approx} \\
0 \\
\vdots \\
\vdots \\
\vdots \\
\dot{0} \\
0\end{array}$ & 0 & 0 & 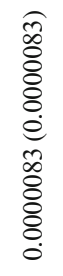 &  & 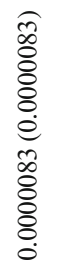 & 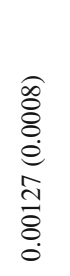 &  & 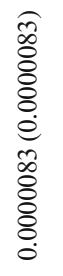 & 0 & & 0 & 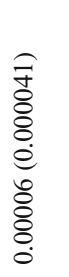 & 0 \\
\hline 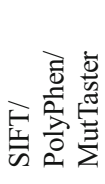 & 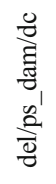 & 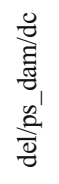 & $\begin{array}{l}\frac{2}{2} \\
\frac{2}{2} \\
\frac{0}{8}\end{array}$ & 䜦 & 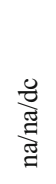 & 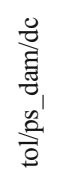 & 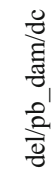 & 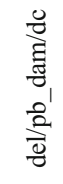 & 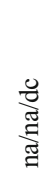 & 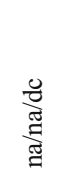 & 苂 & $\begin{array}{l}\stackrel{\Xi}{\Xi} \\
\overparen{\Xi} \\
\overparen{\Xi}\end{array}$ & $\begin{array}{l}0 \\
\text { Dे } \\
\text { 总 }\end{array}$ & 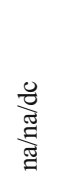 &  & 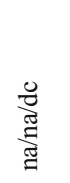 & $\dddot{g}$ & 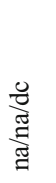 & 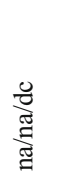 & 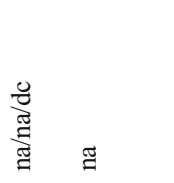 \\
\hline 竞 &  & & 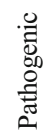 & & & & $\cong$ & & 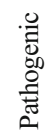 & 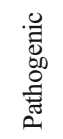 & 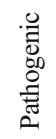 & 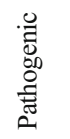 &  & 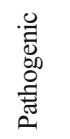 & 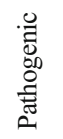 & & & 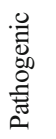 & 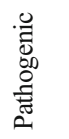 & \\
\hline 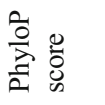 & $\stackrel{\infty}{\beth}$ & $\begin{array}{l}\vec{\infty} \\
\stackrel{+}{+}\end{array}$ & 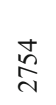 & $\bar{n}$ & ळू & $\stackrel{\hat{\theta}}{n}$ & $\stackrel{\infty}{\infty}$ & 㸃 & $\stackrel{+0}{\stackrel{0}{9}}$ & 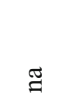 & तิ & $\underset{\stackrel{i}{i}}{\ddot{H}}$ & ڤू. & $\stackrel{\overrightarrow{\mathrm{I}}}{\mathrm{i}}$ & 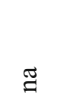 & 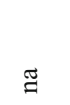 &  & స్ర్ల & 管 & 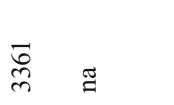 \\
\hline 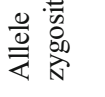 & $\exists$ & $\Xi$ & $\Xi$ & $\nexists$ & $\Xi$ & $\Xi$ & \pm & $\Xi$ & $\Xi_{0}$ & & $\vec{J}_{1}$ & & & $E_{1}$ & & 引 & & है & & $\exists_{1}$ \\
\hline 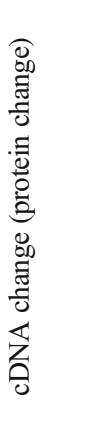 & 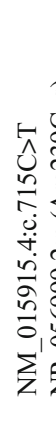 & $1=$ & & 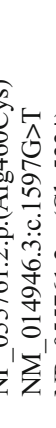 & $\vec{j}$ &  & 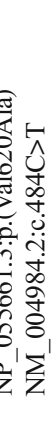 & 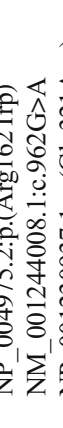 &  & 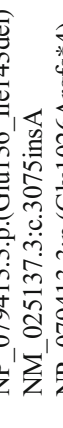 & & 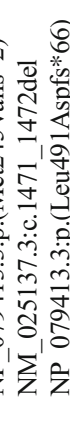 & 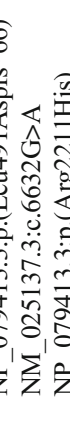 & 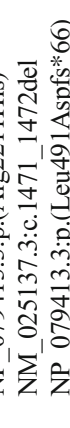 & 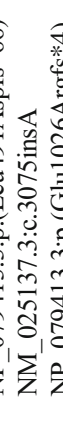 & 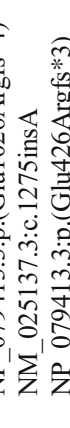 & 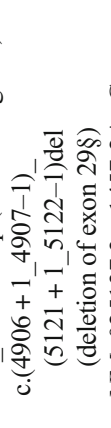 & 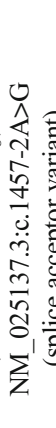 & 年 & 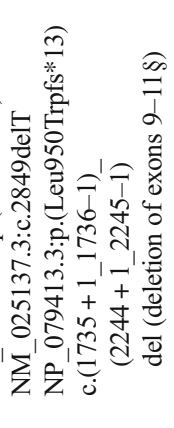 \\
\hline 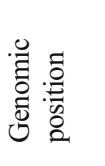 & 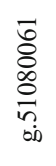 & 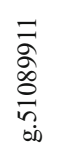 & 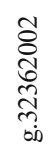 & 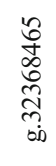 & 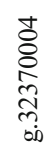 & 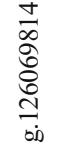 & $\begin{array}{l}\hat{c} \\
\infty \\
o \\
\hat{\alpha} \\
\hat{n} \\
\text { oi }\end{array}$ & 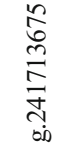 & 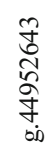 & 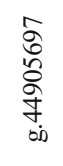 & $\begin{array}{l}\hat{y} \\
\text { d } \\
\text { d } \\
\text { bi }\end{array}$ & 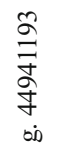 & 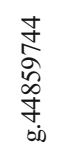 &  & 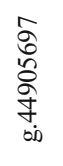 & 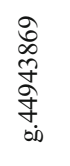 & & 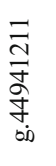 &  & 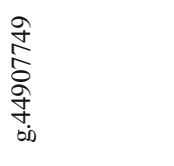 \\
\hline$z$ & \pm & \pm & $\mathrm{N}$ & $\sim$ & N & $\infty$ & $\simeq$ & $N$ & $\stackrel{n}{\sim}$ & & $\stackrel{n}{2}$ & & & $\stackrel{n}{n}$ & & $\because$ & & $\cong$ & & $\cong$ \\
\hline हू & $\overline{\mathrm{Z}}$ & $\bar{Z}$ & 崩 & 氡 & 氛 & 㺼 & 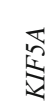 & $\underset{\mathbb{2}}{\mathbb{3}}$ & $\begin{array}{l}\text { స్ } \\
\text { के }\end{array}$ & & స్ర & & & స్ర & & స్ర & & 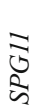 & & స్ \\
\hline 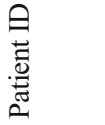 & $\begin{array}{l}\tilde{\delta} \\
8 \\
0 \\
\dot{\omega}\end{array}$ & 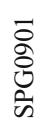 & $\begin{array}{l}\overline{0} \\
0 \\
0 \\
0 \\
\text { in }\end{array}$ & 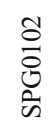 & $\begin{array}{l}\overrightarrow{0} \\
\text { Dे } \\
\text { के }\end{array}$ & 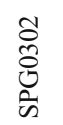 & $\begin{array}{l}\overline{\tilde{\sigma}} \\
\bar{\delta} \\
\text { के }\end{array}$ & $\begin{array}{l}\overrightarrow{0} \\
\overline{0} \\
\dot{\omega}\end{array}$ & $\begin{array}{l}\overrightarrow{0} \\
\dot{0} \\
\text { के }\end{array}$ & & $\begin{array}{l}\text { ठे } \\
\text { Dे } \\
\dot{\omega}\end{array}$ & & & $\begin{array}{l}\tilde{8} \\
\frac{0}{0} \\
\text { के }\end{array}$ & & $\begin{array}{l}\text { مे } \\
\text { डे } \\
\text { के }\end{array}$ & & 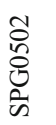 & & $\begin{array}{l}\overrightarrow{0} \\
\text { î̀ } \\
\dot{\omega}\end{array}$ \\
\hline
\end{tabular}


respectively. Two probands had pure HSP, while in one with the nonsense variant, a complicated HSP phenotype with neuropathy as an additional symptom was observed.

\section{WASHC5 (SPG8)}

The WASHC5 missense variants were found in two HSP probands and at least one affected individual within their families. Patient SPG0302 was found to have WASHC5 c. 1859 C $>$ T (p.Val620Ala). The female proband and her affected sibling aged 39 and 37 years at onset - had frontal cortex atrophy. Moreover, in patient SPG0302, white matter and thoracic spinal cord lesions were present. The male proband SPG0403, with WASHC 5 c.647C $>$ T (p.Pro216Leu), presented a complex HSP with dysarthria. His brother with the same variant had intellectual disability in addition to HSP (but he had a verified birth asphyxia - a possible cause of the brain damage).

\section{KIF5A (SPG10)}

Two KIF5A variants were identified in two probands. One of them, KIF5A c. $484 \mathrm{C}>\mathrm{T}$ (p.Arg162Trp), which localised in motor domain of the kinesin protein was present in a proband with pure HSP and onset of symptoms at age 41. The second, KIF5A variant c. $1402 \mathrm{C}>\mathrm{T}$ (p.Arg468Trp), which altered the stalk part of the protein, was identified in a female proband with pyramidal signs, ataxia, dysdiachokinesia, bradykinesia, titubation, ophthalmoparesis and dementia, in whom first symptoms appeared after turning 40. In MRI, marked atrophy of the cerebellum and cerebral cortex (predominantly temporal and parietal) was observed.

KIF1A (SPG30)

A heterozygous KIF1A c.962G>A (p.Gly321Asp) variant, localised in the motor domain of the protein, was found in an AD pedigree. The female proband and her mother had childhood onset, complex hereditary spastic paraplegia and cognitive decline.

\section{Autosomal recessive HSPs}

\section{SPG11 (SPG11)}

The NGS analysis enabled us to identify ten different SPG11 variants (with the ExAC frequency below 0.005) in seven probands. In all of them, the variants were present in both alleles. In the SPG1002 proband, three different variants were detected. In three other patients with single variants found in this study, SPG0103, SPG0301 and SPG0702, the microrearrangements: duplication of exons 28-29, deletions of exons 9-11 and exon 29, respectively, were localised in trans. Five of the variants were frameshift deletions or 


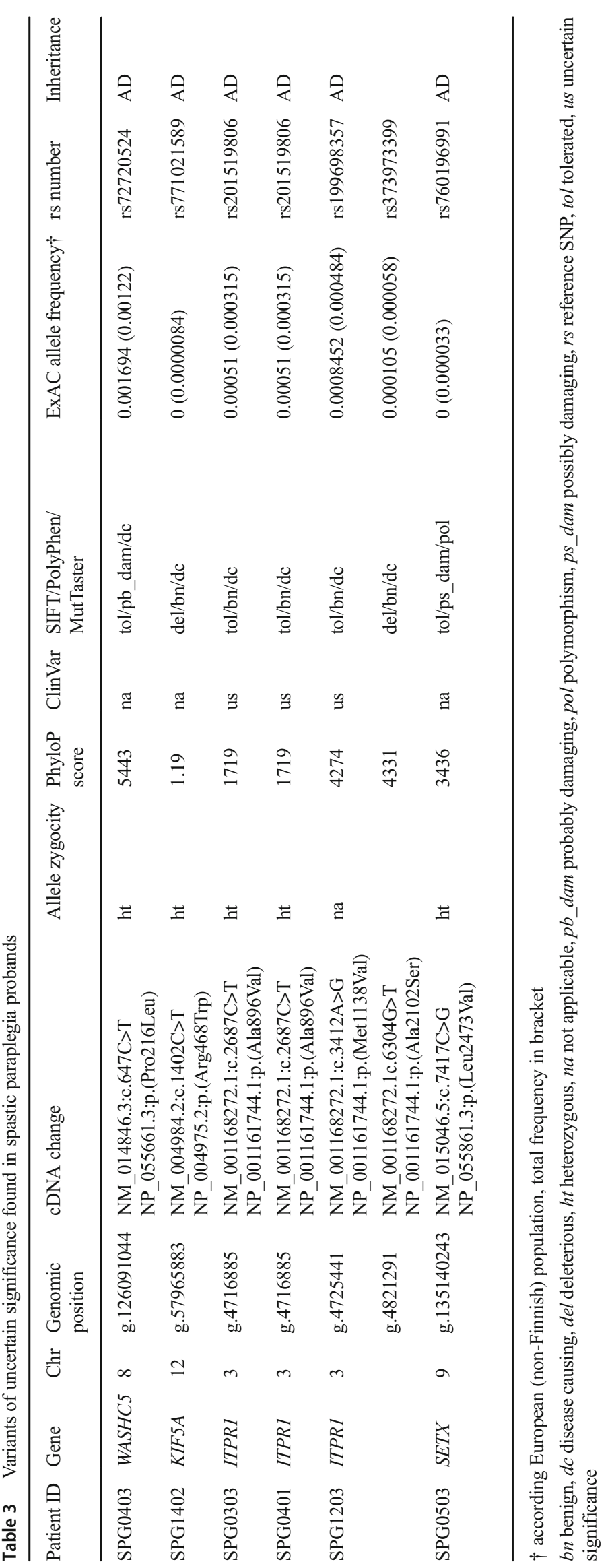


insertions, two were in-frame deletions, one was in the splicesite, one was nonsense and one was a missense change. In SPG1002, the missense variant was identified in cis with the frameshift one.

All of the seven SPG11 probands had a complicated form of HSP and showed cognitive impairment: dysarthria 5/7; dysphagia $2 / 7$; nystagmus $3 / 7$; ophthalmoparesis (horizontal gaze) $2 / 7$; cervical dystonia $1 / 7$ and mild ataxia $3 / 7$. In neuroimaging performed in six probands, thin corpus callosum was found in $5 / 6$, periventricular white matter lesions were found in $4 / 6$, and mild cortical and subcortical atrophy was identified in $2 / 6$. EMG provided evidence of polyneuropathy in three out of five examined probands.

\section{CYP27A1 (CTX)}

In one proband, NGS revealed a homozygous variant, c.379C $>\mathrm{T}$ (p.Arg127Trp) in the CYP27A1 gene, known as pathogenic in cerebrotendinous xanthomatosis (CTX). The carrier status (heterozygosity) was confirmed in the proband's father. The patient, with pyramidal and cerebellar signs, petit mal seizures, bilateral cataract and retinal degeneration in the right eye, was classified as a case of the complicated HSP. Mild cortical and subcortical atrophy were present in brain MRI. Furthermore, in the patient's medical history, vitamin B12 deficiency and nephrolithiasis were documented. To date, neither xanthomas nor other signs characteristic for CTX were not observed in the patient.

\section{Genes with uncertain significance in HSPs}

\section{ITPR1 (GLSP/SCA15/SCA29)}

Three different variants of uncertain significance were identified in the ADHSP patients. ITPR1: c.2687C >T (p.Ala896Val) was identified in seven individuals from two unrelated families with pure HSP. In the SPG1203 proband, two different ITPRI variants (c.3412A $>$ G-p.Met1138Val and c.6304G $>$ Tp.Ala2102Ser) were found. A female patient with weakness and spasticity of her lower limbs, balance disturbances and polyneuropathy had onset of symptoms at age 50 . Genetic testing in her relatives was impossible; however, her family history may indicate $\mathrm{AD}$ inheritance. All the pedigrees and localization of identified ITPRI variants are shown in Fig. 2.

\section{SETX (ALS4/SCAR1)}

One SETX missense variant of uncertain significance, c. $7417 \mathrm{C}>\mathrm{G}$ (p.Leu2473Val), was detected in a 2-year-old proband and the father, who has been affected since childhood. The father's neurological examination showed upper and lower limb weakness and spasticity with increased tendon reflexes and clonus.

\section{Discussion}

Due to heterogeneity, the increasing number of involved genes and varieties of phenotypes (disorders) linked to a single gene, the classification and diagnostics of HSPs are challenging. To overcome these difficulties, different NGS approaches have been applied in a number of studies, mostly targeted sequencing but also whole exome sequencing [13-17]. In the present study, we analysed 30 HSP index cases using the Illumina TruSight ${ }^{\mathrm{TM}}$ One NGS sequencing panel. Bioinfomatic analysis was performed for 132 out of the 4813 genes included in the panel. This methodology allowed us to identify 25 variants in nine genes. The pathogenic and likely pathogenic variants were identified in 16 probands. In five of them, in whom only MLPA technique had been used for microrearrangement searching, we identified three SPAST and two ATL1 variants by NGS. It is an evidence that MLPA is not sufficient for SPG4 testing alone, nonetheless together with NGS is now a standard in diagnostic approach. Less frequent HSP subtypes were identified in a group of patients in whom the SPAST, ATL1 and REEP1 pathogenic variants had been previously excluded. Two different variants were identified in WASHC5 (SPG8, OMIM \#603563, previously known as KIAA0196) and KIF5A (SPG10, OMIM \#604187) genes, both regarded as rare HSP subtypes (approximate frequency 1-2\%) that may be associated with pure or complicated HSP phenotypes [4]. The WASHC5: c.1859T>C (p.Val620Ala) variant has previously been detected in pure HSP patients but has not been reported in either ExAC or the 1000 Genomes projects [18]. The KIF5A:c.484C $>\mathrm{T}$ (p.Arg162Trp) variant has been reported in a three-generation pedigree with spastic paraplegia as a primary symptom [19].

KIF1A is a neuron-specific motor protein involved in intracellular transport along microtubules. Variants in the KIF1A gene have been described in patients with AR hereditary sensory and autonomic neuropathy type 2 (HSAN2, OMIM \#614213) and subtype 30 of the hereditary spastic paraplegia (SPG30, OMIM \#610357) [20-23]. De novo KIF1A variants with $\mathrm{AD}$ transmission have been identified in multiple cases with childhood onset of intellectual disability and a number of neurological signs, such as progressive spastic paraplegia, optic nerve atrophy, peripheral neuropathy and cerebral and/or cerebellar atrophy, have been variously classified as autosomal dominant mental retardation type 9 (MRD9, OMIM\#614255) [24-28] or complicated hereditary spastic paraplegia [25, 29, 30]. Finally, KIF1A mutations have been found in pure HSP subjects [30-32]. In the present study, a dominant KIF1A variant localised in the motor domain of the protein was found in a female proband and her mother with childhood onset complex HSP and cognitive decline. Twenty-three out of 25 heterozygous KIFIA variants (including the present study) alter the highly conserved motor domain of the protein. However, two out of four variants responsible for recessive HSP and any of the variants identified in HSAN2 are localised in the motor 
$\mathrm{IP}_{3}$-binding domain

Coupling/regulatory domain

Transmembranne C-terminal

$* \diamond$

domain

tail

a

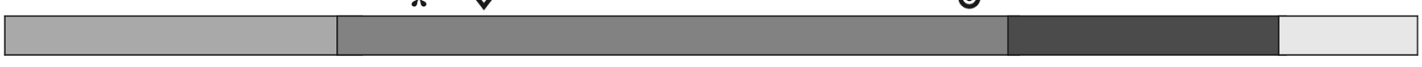

IRBIT

CARP

b


SPG0401* ITPRI: c. $2687 \mathrm{C}>\mathrm{T}$

$$
\begin{aligned}
& \text { SPG1203 }^{\diamond} \text { ITPR } 1: \text { c. } 3412 \mathrm{~A}>\mathrm{G}(;) 6304 \mathrm{G}>\mathrm{T} \\
& \text { p.(Met1138Val)(;)(Ala2102Ser) }
\end{aligned}
$$

Fig. 2 A ITPR1 protein scheme. Localization of three identified variants interrupting coupling/regulatory domain is showed by: “*”, p.(Ala896Val); “», p.(Met1138Val); “•”, p.(Ala2102Ser). IRBIT, Inositol 1,4,5-trisphosphate $\left(\mathrm{IP}_{3}\right)$ receptor binding domain; CARP, Carbonic anhydrase-related protein (CA8) binding domain. B Pedigrees of three families with ITPRI variants. Families SPG0303 and

domain. This suggests that localization of the KIF1A variants within the gene is not adequate evidence for phenotype transmission. Moreover, the latest data indicate that dominant conditions, including ADHSP, linked with KIFIA variants are more frequent than recessive ones.

SPG11 (OMIM \#604360) is the only known recessive HSP subtype identified in this study. Contrary to other studies, we have not detected any affected patient with CYP7B1 (SPG5, OMIM \#270800) or SPG7 (SPG7, OMIM \#607259) mutations, or any mutation carriers [13-17, 32]. Moreover, variants in ZFYVE26 (SPG15, OMIM \#270700), which occur with frequency below 0.005 in the ExAC database, were not detected in our cohort.

In addition to the recessive variants, in one case, we detected a homozygous variant in the CYP27A1 gene. Pathogenic variants in the cytochrome $\mathrm{P} 450$ CYP27A1 gene result in the production
SPG0401 are marked with "*” which indicates ITPR1: c. $2687 \mathrm{C}>\mathrm{T}$ (p.Ala896Val); family SPG1203 is marked with "»" and "•" which indicate ITPR 1: c.3412A>G (p.Met1138Val) and c.6304G > T (p.Ala2102Ser), respectively. The "+" points out family members, in whom the DNA samples were tested; "-“affected individuals without DNA testing

of a defective sterol 27-hydrolase enzyme and have been linked with cerebrotendinous xanthomatosis (CTX) (OMIM \#213700). Clinical manifestation of CTX includes neurological dysfunction (e.g. cerebellar ataxia, pyramidal signs, and seizures), cataracts, tendon xanthomas and chronic diarrhoea [33, 34]. However, some atypical presentation of symptoms may occur. For example, Verrips et al. described seven patients with CYP27A1 variants and slowly progressive spinal cord syndrome classified as spinal xanthomatosis. Moreover, similar to our case, all of the patients presented pyramidal signs, and in five of them, spinal cord white matter lesion have been demonstrated. Six out of seven cases studied by Verrips et al. did not have tendon xanthomas [35]. Patients with CYP27A1 variants affected with pure and complicated HSP but without xanthomas were also described by Burguez et al. and Nicholls et al. [15, 36]. These findings suggest that patients with $C Y P 27 A 1$ variants may 
present the broader clinical spectrum including HSP phenotype, nonetheless the lack of the typical symptoms of CTX, especially xantomas, should not exclude the investigation of CYP27A1 gene mutations.

Variants of uncertain significance within ITPRI and SETX genes were detected in four cases. ITPRI variants have already been described as possibly corresponding to four different phenotypes: multi-exon deletions in ITPR1 gene to spinocerebellar ataxia type 15 (SCA15, OMIM \#606658), single nucleotide variants to spinocerebellar ataxia type 29 (SCA29, OMIM \#117360) or ataxic cerebral palsy (Ataxic $\mathrm{CP}$ ), and the truncated and splice-site variants in Gillespie Syndrome (GLSP, OMIM \#206700) also presented ataxia and balance disturbances [37-42]. ITPR 1 encodes a homotetramer calcium channel protein that modulates intracellular calcium signalling. Its primary structure consists of three major domains [43]. In this study the ITPRI c. $2687 \mathrm{C}>\mathrm{T}$ (p.Ala896Val) variant was detected in two unrelated families and segregates with pure HSP phenotype in seven cases. We also identified two different ITPRI variants in a patient with pyramidal signs and polyneuropathy. Although the three described variants were reported in the ExAC database, their frequency was lower than 0.005 (Table 2b). The relatively mild HSP symptoms in our patients were first observed in adulthood i.e. the age of onset was not optimal for control studies. The segregation data in the families with c. $2687 \mathrm{C}>\mathrm{T}$ (p.Ala896Val) supports its pathogenicity; however, according to the ACMGG\&AMP guidelines, this is not adequate evidence to classify it as a pathogenic/probably pathogenic variant. Variants identified in the present study are localised in the coupling-domain and comprise the first report assigning ITPRI variants to HSP.

A variant classified as of uncertain significance was also found in the senataxin gene. SETX variants are responsible for AR spinocerebellar ataxia (SCAR1) and AD amyotrophic lateral sclerosis (ALS4) [44-48]. The heterozygous variant of the SETX gene has also been described as a cause of hereditary motor neuropathy (dHMN) $[49,50]$. Taniguchi et al. reported a family with a SETX variant misdiagnosed as a hereditary spastic paraplegia [51]. The mentioned variant (SETX:c.8C $>$ T) was localised in the N-terminal end of the protein, different than the SETX: c. $7417 \mathrm{C}>\mathrm{G}$ (p.Leu2473 Val), altering the C-terminal part of the protein, which was identified during our study in father and son with pure HSP. It is localised in the region of the helicase domain, where known pathogenic variants correlated with ALS4 and SCAR1 phenotypes had been reported as well [52].

Although the molecular investigation of rare heterogenic disorders, such as hereditary spastic paraplegias, will soon be based on massive NGS technology, their molecular aetiology assessment still remains challenging. Two major difficulties to face at present are: (1) interpretation of the detected variants (pathogenic vs benign) and (2) classification of the identified variant and its association with a specific disease. Unified and reliable sequence variants interpretation guidelines were developed by the American College of Medical Genetics and Genomics and the Association for Molecular Pathology. Each rare or novel variant should be evaluated in a patient's and family's history context, and physical examination and previous differential diagnosis should be performed. Such clinical evaluation is supportive during the process of variants classification as disease-causing, incidental or benign findings [12]. Variants classified as pathogenic but also likely pathogenic have sufficient evidence to be used in genetic counselling and clinical decision-making. In contrast, variants of uncertain significance need further investigation that may result in their reclassification [12].

Implementing NGS technologies in clinical practice also brings problems due to the genotype-phenotype correlation and variants' classification. The classification systems were designed according to a predominant disease phenotype and/ or a mode of inheritance. Currently, various genes corresponding to numerous complex phenotypes, such as spinocerebellar ataxias, spastic paraplegias and amyotrophic lateral sclerosis, are associated with $S P G 7, S P G 11, P N P L A 6, K I F 1 C$ and $S E T X$, and they may be inherited as both autosomal dominant and recessive traits (KIF1A, REEP2, AFG3L2, SETX). In clinical practice, it becomes problematic whether the identified gene variant should be classified as corresponding to a new phenotype or if it "fits" the patient's genotype consistent with the previous clinical diagnosis. Synofzik et al. proposed introducing the unbiased modular phenotyping approach to replace the ataxias and hereditary spastic paraplegia classification [6]. In parallel, we also recommend simultaneously testing and analysing the HSP, SCA and ALS genes due to their overlapping phenotype and common cellular pathways involved.

In this paper, we report 24 different variants of nine genes in HSP patients. Seven of the variants are novel. They were classified according to the ACMGG\&AMP guidelines, and nine were classified as pathogenic, nine as likely pathogenic and six as of uncertain significance. Among nine analysed genes, five have already been known as directly associated with HSP. NGS testing revealed genetic variants in 22 out of 30 tested families. Altogether with the previous study [8], seven different HSP subtypes have been diagnosed in the Polish group of patients to date. Our data also support the evidence that KIF1A (SPG30) variants are more frequent in patients with ADHSP, although they were primarily identified as ARHSP. Moreover, we believe that CYP27A1 variants should be considered to be complicated HSP phenotype cases, as well.

The overlapping phenotypes of HSP, SCA and ALS are associated with multiple genes; therefore, NGS-based screening provides the best comprehensive genetic diagnostic approach. The most challenging interpretation of the novel variants requires the entire body of clinical and molecular evidence available in the entire studied group of patients sharing a defined spectrum of clinical signs. 
Acknowledgments We appreciate all patients and their families, as well as medical doctors from the Institute of Mother and Child in Warsaw (Poland): Prof. Elzbieta Szczepanik, Dr. Ewa Obersztyn and Prof. Hanna Mierzewska.

Funding information This study was financed by Operational Programme Innovative Economy, Activity 1.1.2 (UDA-POIG.01.01.0214-051/09-00) and the Ministry of Education and Science internal programme: "Designing the diagnostic tool for genetic analysis of the hereditary spastic paraplegia using Next Generation Sequencing - NGS."

Open Access This article is distributed under the terms of the Creative Commons Attribution 4.0 International License (http:// creativecommons.org/licenses/by/4.0/), which permits unrestricted use, distribution, and reproduction in any medium, provided you give appropriate credit to the original author(s) and the source, provide a link to the Creative Commons license, and indicate if changes were made.

Publisher's note Springer Nature remains neutral with regard to jurisdictional claims in published maps and institutional affiliations.

\section{References}

1. Parodi L, Fenu S, Stevanin G, Durr A (2017) Hereditary spastic paraplegia: more than an upper motor neuron disease. Rev Neurol (Paris) 173(5):352-360

2. Estrada-Cuzcano A, Martin S, Chamova T, Synofzik M, Timmann D, Holemans T, Andreeva A, Reichbauer J, De Rycke R, Chang DI et al (2017) Loss-of-function mutations in the ATP13A2/PARK9 gene cause complicated hereditary spastic paraplegia (SPG78). Brain 140(2):287-305

3. Rydning SL, Backe PH, Sousa MML, Iqbal Z, Oye AM, Sheng Y, Yang M, Lin X, Slupphaug G, Nordenmark TH et al (2017) Novel UCHL1 mutations reveal new insights into ubiquitin processing. Hum Mol Genet 26(6):1031-1040

4. Klebe S, Stevanin G, Depienne C (2015) Clinical and genetic heterogeneity in hereditary spastic paraplegias: from SPG1 to SPG72 and still counting. Rev Neurol (Paris) 171(6-7):505-530

5. Ruano L, Melo C, Silva MC, Coutinho P (2014) The global epidemiology of hereditary ataxia and spastic paraplegia: a systematic review of prevalence studies. Neuroepidemiology 42(3):174-183

6. Synofzik M, Schule R (2017) Overcoming the divide between ataxias and spastic paraplegias: shared phenotypes, genes, and pathways. Mov Disord 32(3):332-345

7. Sulek A, Elert E, Rajkiewicz M, Zdzienicka E, Stepniak I, Krysa W, Zaremba J (2013) Screening for the hereditary spastic paraplaegias SPG4 and SPG3A with the multiplex ligation-dependent probe amplification technique in a large population of affected individuals. Neurol Sci 34(2):239-242

8. Elert-Dobkowska E, Stepniak I, Krysa W, Rajkiewicz M, Rakowicz M, Sobanska A, Rudzinska M, Wasielewska A, Pilch J, Kubalska J, Lipczynska-Lojkowska W, Kulczycki J, Kurdziel K, Sikorska A, Beetz C, Zaremba J, Sulek A (2015) Molecular spectrum of the SPAST, ATL1 and REEP1 gene mutations associated with the most common hereditary spastic paraplegias in a group of Polish patients. J Neurol Sci 359(1-2):35-39

9. Gunther S, Elert-Dobkowska E, Soehn AS, Hinreiner S, Yoon G, Heller R, Hellenbroich Y, Hubner CA, Ray PN, Hehr U et al (2016) High frequency of pathogenic rearrangements in SPG11 and extensive contribution of mutational hotspots and founder alleles. Hum Mutat 37(7):703-709

10. Elert-Dobkowska E, Stepniak I, Rajkiewicz M, Krysa W, Rakowicz M, Hoffman-Zacharska D, Lipczyńska-Lojkowska W, Zaremba J, Sulek A (2014) Familial 15q11.2 microdeletions are not fully penetrant in two cases with hereditary spastic paraplegia and dysmorphic features. J Genet Syndr Gene Ther 5:247. https://doi.org/ 10.4172/2157-7412.1000247

11. Fink JK, Heiman-Patterson T, Bird T, Cambi F, Dube MP, Figlewicz DA, Fink JK, Haines JL, Heiman-Patterson T, Hentati A et al (1996) Hereditary spastic paraplegia: advances in genetic research. Hereditary spastic paraplegia working group. Neurology 46(6):1507-1514

12. Richards S, Aziz N, Bale S, Bick D, Das S, Gastier-Foster J, Grody WW, Hegde M, Lyon E, Spector E et al (2015) Standards and guidelines for the interpretation of sequence variants: a joint consensus recommendation of the American College of Medical Genetics and Genomics and the Association for Molecular Pathology. Genet Med 17(5):405-424

13. Chrestian N, Dupre N, Gan-Or Z, Szuto A, Chen S, Venkitachalam A, Brisson JD, Warman-Chardon J, Ahmed S, Ashtiani S et al (2017) Clinical and genetic study of hereditary spastic paraplegia in Canada. Neurol Genet 3(1):e122

14. Kara E, Tucci A, Manzoni C, Lynch DS, Elpidorou M, Bettencourt C, Chelban V, Manole A, Hamed SA, Haridy NA, Federoff M, Preza E, Hughes D, Pittman A, Jaunmuktane Z, Brandner S, Xiromerisiou G, Wiethoff S, Schottlaender L, Proukakis C, Morris H, Warner T, Bhatia KP, Korlipara LVP, Singleton AB, Hardy J, Wood NW, Lewis PA, Houlden H (2016) Genetic and phenotypic characterization of complex hereditary spastic paraplegia. Brain 139(Pt 7):1904-1918

15. Burguez D, Polese-Bonatto M, Scudeiro LAJ, Bjorkhem I, Schols L, Jardim LB, Matte U, Saraiva-Pereira ML, Siebert M, Saute JAM (2017) Clinical and molecular characterization of hereditary spastic paraplegias: a next-generation sequencing panel approach. J Neurol Sci 383:18-25

16. Lynch DS, Koutsis G, Tucci A, Panas M, Baklou M, Breza M, Karadima G, Houlden H (2016) Hereditary spastic paraplegia in Greece: characterisation of a previously unexplored population using next-generation sequencing. Eur J Hum Genet 24(6):857-863

17. Kumar KR, Blair NF, Vandebona H, Liang C, Ng K, Sharpe DM, Grunewald A, Golnitz U, Saviouk V, Rolfs A et al (2013) Targeted next generation sequencing in SPAST-negative hereditary spastic paraplegia. J Neurol 260(10):2516-2522

18. Jahic A, Kreuz F, Zacher P, Fiedler J, Bier A, Reif S, Rieger M, Kruger S, Beetz C, Plaschke J (2014) A novel strumpellin mutation and potential pitfalls in the molecular diagnosis of hereditary spastic paraplegia type SPG8. J Neurol Sci 347(1-2):372-374

19. Carosi L, Lo Giudice T, Di Lullo M, Lombardi F, Babalini C, Gaudiello F, Marfia GA, Massa R, Kawarai T, Orlacchio A (2015) Hereditary spastic paraplegia: a novel mutation and expansion of the phenotype variability in SPG10. J Neurol Neurosurg Psychiatry 86(6):702-704

20. Riviere JB, Ramalingam S, Lavastre V, Shekarabi M, Holbert S, Lafontaine J, Srour M, Merner N, Rochefort D, Hince P et al (2011) $\mathrm{KIF} 1 \mathrm{~A}$, an axonal transporter of synaptic vesicles, is mutated in hereditary sensory and autonomic neuropathy type 2 . Am J Hum Genet 89(2):219-230

21. Klebe S, Lossos A, Azzedine H, Mundwiller E, Sheffer R, Gaussen M, Marelli C, Nawara M, Carpentier W, Meyer V, Rastetter A, Martin E, Bouteiller D, Orlando L, Gyapay G, el-Hachimi KH, Zimmerman B, Gamliel M, Misk A, Lerer I, Brice A, Durr A, Stevanin G (2012) KIF1A missense mutations in SPG30, an autosomal recessive spastic paraplegia: distinct phenotypes according to the nature of the mutations. Eur J Hum Genet 20(6):645-649

22. Krenn M, Zulehner G, Hotzy C, Rath J, Stogmann E, Wagner M, Haack TB, Strom TM, Zimprich A, Zimprich F (2017) Hereditary spastic paraplegia caused by compound heterozygous mutations outside the motor domain of the KIF1A gene. Eur J Neurol 24(5):741-747

23. Erlich Y, Edvardson S, Hodges E, Zenvirt S, Thekkat P, Shaag A, Dor T, Hannon GJ, Elpeleg O (2011) Exome sequencing and diseasenetwork analysis of a single family implicate a mutation in KIF1A in hereditary spastic paraparesis. Genome Res 21(5):658-664 
24. Lee JR, Srour M, Kim D, Hamdan FF, Lim SH, Brunel-Guitton C, Decarie JC, Rossignol E, Mitchell GA, Schreiber A et al (2015) De novo mutations in the motor domain of KIF1A cause cognitive impairment, spastic paraparesis, axonal neuropathy, and cerebellar atrophy. Hum Mutat 36(1):69-78

25. Cheon CK, Lim SH, Kim YM, Kim D, Lee NY, Yoon TS, Kim NS, Kim E, Lee JR (2017) Autosomal dominant transmission of complicated hereditary spastic paraplegia due to a dominant negative mutation of KIF1A, SPG30 gene. Sci Rep 7(1):12527

26. Hamdan FF, Gauthier J, Araki Y, Lin DT, Yoshizawa Y, Higashi K, Park AR, Spiegelman D, Dobrzeniecka S, Piton A, Tomitori H, Daoud H, Massicotte C, Henrion E, Diallo O, S2D Group, Shekarabi M, Marineau C, Shevell M, Maranda B, Mitchell G, Nadeau A, D'Anjou G, Vanasse M, Srour M, Lafrenière RG, Drapeau P, Lacaille JC, Kim E, Lee JR, Igarashi K, Huganir RL, Rouleau GA, Michaud JL (2011) Excess of de novo deleterious mutations in genes associated with glutamatergic systems in nonsyndromic intellectual disability. Am J Hum Genet 88(3):306-316

27. Ohba C, Haginoya $K$, Osaka $H$, Kubota $K$, Ishiyama A, Hiraide T, Komaki H, Sasaki M, Miyatake S, Nakashima M, Tsurusaki Y, Miyake N, Tanaka F, Saitsu H, Matsumoto N (2015) De novo KIF1A mutations cause intellectual deficit, cerebellar atrophy, lower limb spasticity and visual disturbance. J Hum Genet 60(12):739-742

28. Esmaeeli Nieh S, Madou MR, Sirajuddin M, Fregeau B, McKnight D, Lexa K, Strober J, Spaeth C, Hallinan BE, Smaoui N et al (2015) De novo mutations in KIF1A cause progressive encephalopathy and brain atrophy. Ann Clin Transl Neurol 2(6):623-635

29. Hotchkiss L, Donkervoort S, Leach ME, Mohassel P, BharuchaGoebel DX, Bradley N, Nguyen D, Hu Y, Gurgel-Giannetti J, Bonnemann CG (2016) Novel de novo mutations in KIF1A as a cause of hereditary spastic paraplegia with progressive central nervous system involvement. J Child Neurol 31(9):1114-1119

30. Citterio A, Arnoldi A, Panzeri E, Merlini L, D'Angelo MG, Musumeci O, Toscano A, Bondi A, Martinuzzi A, Bresolin N et al (2015) Variants in KIF1A gene in dominant and sporadic forms of hereditary spastic paraparesis. J Neurol 262(12):2684-2690

31. Ylikallio E, Kim D, Isohanni P, Auranen M, Kim E, Lonnqvist T, Tyynismaa H (2015) Dominant transmission of de novo KIF1A motor domain variant underlying pure spastic paraplegia. Eur J Hum Genet 23(10):1427-1430

32. Iqbal Z, Rydning SL, Wedding IM, Koht J, Pihlstrom L, Rengmark AH, Henriksen SP, Tallaksen CM, Toft M (2017) Targeted high throughput sequencing in hereditary ataxia and spastic paraplegia. PLoS One 12(3):e0174667

33. Salen G, Steiner RD (2017) Epidemiology, diagnosis, and treatment of cerebrotendinous xanthomatosis (CTX). J Inherit Metab Dis 40(6):771-781

34. Kapas I, Katko M, Harangi M, Paragh G, Balogh I, Koczi Z, Regelsberger G, Molnar MJ, Kovacs GG (2014) Cerebrotendinous xanthomatosis with the c.379C>T (p.R127W) mutation in the CYP27A1 gene associated with premature age-associated limbic tauopathy. Neuropathol Appl Neurobiol 40(3):345-350

35. Verrips A, Nijeholt GJ, Barkhof F, Van Engelen BG, Wesseling P, Luyten JA, Wevers RA, Stam J, Wokke JH, van den Heuvel LP et al (1999) Spinal xanthomatosis: a variant of cerebrotendinous xanthomatosis. Brain 122(Pt 8):1589-1595

36. Nicholls Z, Hobson E, Martindale J, Shaw PJ (2015) Diagnosis of spinal xanthomatosis by next-generation sequencing: identifying a rare, treatable mimic of hereditary spastic paraparesis. Pract Neurol 15(4):280-283

37. Synofzik M, Beetz C, Bauer C, Bonin M, Sanchez-Ferrero E, Schmitz-Hubsch T, Wullner U, Nagele T, Riess O, Schols L, Bauer P (2011) Spinocerebellar ataxia type 15: diagnostic assessment, frequency, and phenotypic features. J Med Genet 48(6):407-412

38. Marelli C, van de Leemput J, Johnson JO, Tison F, ThauvinRobinet C, Picard F, Tranchant C, Hernandez DG, Huttin B,
Boulliat J, Sangla I, Marescaux C, Brique S, Dollfus H, Arepalli S, Benatru I, Ollagnon E, Forlani S, Hardy J, Stevanin G, Dürr A, Singleton A, Brice A (2011) SCA15 due to large ITPR1 deletions in a cohort of 333 white families with dominant ataxia. Arch Neurol 68(5):637-643

39. van de Leemput J, Chandran J, Knight MA, Holtzclaw LA, Scholz S, Cookson MR, Houlden H, Gwinn-Hardy K, Fung HC, Lin X, Hernandez D, Simon-Sanchez J, Wood NW, Giunti P, Rafferty I, Hardy J, Storey E, Gardner RJMK, Forrest SM, Fisher EMC, Russell JT, Cai H, Singleton AB (2007) Deletion at ITPR1 underlies ataxia in mice and spinocerebellar ataxia 15 in humans. PLoS Genet 3(6):e108

40. Das J, Lilleker J, Shereef H, Ealing J (2017) Missense mutation in the ITPR1 gene presenting with ataxic cerebral palsy: description of an affected family and literature review. Neurol Neurochir Pol 51(6):497-500

41. Huang L, Chardon JW, Carter MT, Friend KL, Dudding TE, Schwartzentruber J, Zou R, Schofield PW, Douglas S, Bulman DE, Boycott KM (2012) Missense mutations in ITPR1 cause autosomal dominant congenital nonprogressive spinocerebellar ataxia. Orphanet J Rare Dis 7:67

42. Barresi S, Niceta M, Alfieri P, Brankovic V, Piccini G, Bruselles A, Barone MR, Cusmai R, Tartaglia M, Bertini E, Zanni G (2017) Mutations in the IRBIT domain of ITPR1 are a frequent cause of autosomal dominant nonprogressive congenital ataxia. Clin Genet 91(1):86-91

43. Sugawara T, Hisatsune C, Le TD, Hashikawa T, Hirono M, Hattori M, Nagao S, Mikoshiba K (2013) Type 1 inositol trisphosphate receptor regulates cerebellar circuits by maintaining the spine morphology of Purkinje cells in adult mice. J Neurosci 33(30):12186-12196

44. Chen YZ, Bennett CL, Huynh HM, Blair IP, Puls I, Irobi J, Dierick I, Abel A, Kennerson ML, Rabin BA, Nicholson GA, AuerGrumbach M, Wagner K, de Jonghe P, Griffin JW, Fischbeck KH, Timmerman V, Cornblath DR, Chance PF (2004) DNA/RNA helicase gene mutations in a form of juvenile amyotrophic lateral sclerosis (ALS4). Am J Hum Genet 74(6):1128-1135

45. Moreira MC, Klur S, Watanabe M, Nemeth AH, Le Ber I, Moniz JC, Tranchant C, Aubourg P, Tazir M, Schols L et al (2004) Senataxin, the ortholog of a yeast RNA helicase, is mutant in ataxia-ocular apraxia 2. Nat Genet 36(3):225-227

46. Asaka T, Yokoji H, Ito J, Yamaguchi K, Matsushima A (2006) Autosomal recessive ataxia with peripheral neuropathy and elevated AFP: novel mutations in SETX. Neurology 66(10):1580-1581

47. Airoldi G, Guidarelli A, Cantoni O, Panzeri C, Vantaggiato C, Bonato S, Grazia D'Angelo M, Falcone S, De Palma C, Tonelli A et al (2010) Characterization of two novel SETX mutations in AOA2 patients reveals aspects of the pathophysiological role of senataxin. Neurogenetics 11(1):91-100

48. Kenna KP, McLaughlin RL, Byrne S, Elamin M, Heverin M, Kenny EM, Cormican P, Morris DW, Donaghy CG, Bradley DG et al (2013) Delineating the genetic heterogeneity of ALS using targeted high-throughput sequencing. J Med Genet 50(11):776-783

49. Rossor AM, Kalmar B, Greensmith L, Reilly MM (2012) The distal hereditary motor neuropathies. J Neurol Neurosurg Psychiatry 83(1):6-14

50. Drew AP, Zhu D, Kidambi A, Ly C, Tey S, Brewer MH, AhmadAnnuar A, Nicholson GA, Kennerson ML (2015) Improved inherited peripheral neuropathy genetic diagnosis by wholeexome sequencing. Mol Genet Genomic Med 3(2):143-154

51. Taniguchi T, Hokezu Y, Okada T, Ishibashi M, Hashiguchi A, Matsuura E, Takashima H (2017) A amyotrophic lateral sclerosis (ALS) 4 family misdiagnosed as hereditary spastic paraplegia-a case report. Rinsho Shinkeigaku 57(11):685-690

52. Bennett CL, La Spada AR (2015) Unwinding the role of senataxin in neurodegeneration. Discov Med 19(103):127-136 